\title{
Ferromagnetic phase in the polarized two-species bosonic Hubbard Model
}

\author{
Kalani Hettiarachchilage, ${ }^{1,2}$ Valéry G. Rousseau, ${ }^{1,2}$ Ka-Ming Tam, ${ }^{1,2}$ Mark Jarrell, ${ }^{1,2}$ and Juana Moreno ${ }^{1,2}$ \\ ${ }^{1}$ Department of Physics and Astronomy, Louisiana State University, Baton Rouge, Louisiana 70803, USA \\ ${ }^{2}$ Center for Computation and Technology, Louisiana State University, Baton Rouge, LA 70803, USA
}

(Dated: August 13, 2018)

\begin{abstract}
We recently studied a doped two-dimensional bosonic Hubbard model with two hard-core species, with different masses, using quantum Monte Carlo simulations [Phys. Rev. B 88, 161101(R) (2013)]. Upon doping away from half-filling, we find several distinct phases, including a phase-separated ferromagnet with Mott behavior for the heavy species and both Mott insulating and superfluid behaviors for the light species. Introducing polarization, an imbalance in the population between species, we find a fully phase-separated ferromagnet. This phase exists for a broad range of temperatures and polarizations. By using finite size scaling of the susceptibility, we find a critical exponent which is consistent with the two-dimensional Ising universality class. Significantly, since the global entropy of this phase is higher than that of the ferromagnetic phase with single species, its experimental observation in cold atoms may be feasible.
\end{abstract}

PACS numbers: $02.70 . \mathrm{Uu}, 05.30 . \mathrm{Jp}$

\section{INTRODUCTION}

One of the frontiers of condensed matter physics is the study of competing quantum phases such as coexistent and inhomogeneous phases, quantum criticality, and secondary ordered phases close to quantum critical points $\underline{\underline{1}} \underline{\underline{6}}$ These exotic phenomena in strongly correlated systems occur due to the competition and cooperation between the spin, charge, lattice, and orbital degrees of freedom $\underline{\underline{7}}$ Unfortunately, it is often difficult to differentiate the effect of these degrees of freedom in real materials. However, the advance of optical lattice experiments provides a tantalizing opportunity to study competing phases via controlled external parameters $\stackrel{8}{\underline{8}-10}$

The experimental tunability of Hamiltonian parameters using laser and magnetic fields $\underline{11}, 12$ allows the realization of strongly correlated model Hamiltonians. The realization of the Bose-Hubbard model using ultra-cold atoms on optical lattices 13 has led to the observation 14,15 of the Mott-insulator to superfluid phase transition. The Mott insulator phase is characterized by commensurate occupations, gapped excitations and incompressibility in the strong coupling regime. The superfluid phase is characterized by Bose-Einstein condensation, gapless excitations and finite compressibility in the weak coupling regime.

This success has spurred interest in mixtures of atoms which can give rise to even more interesting and complex phases. These include mixtures of bosonic and fermionic atoms $\underline{16}-19$ (a Bose-Fermi mixture) and mixtures of two different bosonic species (a Bose-Bose mixture) ${ }^{20}-22$ Moreover, experimental studies of ${ }^{85} R b_{-}{ }^{87} R b$, ${ }^{87} \mathrm{Rb}^{41} \mathrm{~K},{ }^{6} \mathrm{Li}^{40} \mathrm{~K}$ and different alkaline earth mixtures in optical lattices $23-25$ have motivated theoretical studies of the two species Bose-Hubbard model $\stackrel{26-34}{\underline{34}}$ The zerotemperature phase diagram of the two-dimensional, twospecies, hard-core bosonic Hubbard model has been studied at half-filling using a combination of mean field and variational methods $\stackrel{26}{=}$ and by means of quantum Monte
Carlo simulations $\frac{27}{2}$ The rich phase diagram found at half-filling in these studies shows ordered Mott insulating phases including anti-ferromagnetic and super-counterfluid phases in the strong interaction limit. On the other hand, superfluid and antiferromagnetic/superfluid phases are found in the weak interaction limit $\underline{26-30}$ Recently, we have included doping dependence as a control parameter to study this model using quantum Monte Carlo simulations. We found several distinct phases including a normal liquid at higher temperatures, an antiferromagnetically ordered Mott insulator, and a region of coexistent antiferromagnetic and superfluid order near half-filling. ${ }^{34}$ We also reported a small dome containing a phase-separated ferromagnetic phase away from halffilling at zero polarization.

Though the realization of quantum magnetic phases has gained significant attention, the prominent experimental challenge is to reach the low temperatures and entropies needed to observe these phases. Several different experimental techniques have been proposed to reach such low entropies $\stackrel{35-37}{-3 n t e r e s t i n g l y, ~ a ~ B o s e-F e r m i ~ m i x-~}$ ture may be used to squeeze the entropy of a Fermi gas into the surrounding Bose gas $\underline{38}$ This can leave a low entropy heavy Fermi gas by evaporating the entropy absorbed by the light Bose gas. The relatively high global entropy of the phase-separated ferromagnetic phase we found away from half-filling in the two species Hubbard model ${ }^{34}$ suggests that this ferromagnet should be easier to access experimentally.

In the experimental setup of boson-boson mixtures, the two species are not always perfectly balanced 21,39 The evaporative cooling leads to net losses of one of the species, due to the difference in the effective depth of the traps. This can be adjusted by loading different number of atoms for different species into the trap $\underline{\underline{39}}$ This procedure can also be used to set an imbalance amount of atoms for the two species. Most of the previous theoretical or numerical studies on the two-species Bose-Hubbard model do not directly address this imbalance in the ex- 
perimental conditions.

In this paper, we explore the extent of the phaseseparated ferromagnetic phase as a function of finite polarization, i.e., with a different population for each species. When the polarization is positive (more of the light than heavy particles) we find a larger region of the ferromagnetic phase-separated order, with higher transition temperatures and greater extent in doping. Since this ferromagnetic phase exists for a broad range of sufficiently high temperatures and polarizations together with high global entropies, experimental observation in cold atoms may be achievable.

This manuscript is organized as follows. In section II we describe our model and method. The density versus polarization phase diagram at low temperature is studied in section III. In section IV] we discuss the temperature versus polarization phase diagram along an optimal superfluid or maximum ferromagnetic phase line. The momentum distribution of the ferromagnetic and superfluid phases are presented in section $\mathrm{V}$. In section VI we calculate the entropy of the ferromagnetic, antiferromagnetic and superfluid phases. Finally we conclude in section VII

\section{MODEL AND METHOD}

The Hamiltonian for the two-species Hubbard model with hard-core heavy, $a$, and light, $b$, bosons confined on a two-dimensional square lattice takes the form:

$$
\begin{aligned}
\hat{\mathcal{H}}= & -t_{a} \sum_{\langle i, j\rangle}\left(a_{i}^{\dagger} a_{j}+H . c .\right) \\
& -t_{b} \sum_{\langle i, j\rangle}\left(b_{i}^{\dagger} b_{j}+H . c .\right)+U_{a b} \sum_{i} \hat{n}_{i}^{a} \hat{n}_{i}^{b},
\end{aligned}
$$

where $a_{i}^{\dagger}\left(b_{i}^{\dagger}\right)$ and $a_{i}\left(b_{i}\right)$ are the creation and annihilation operators of hard-core bosons $a$ and $(b)$, respectively, with number operators $\hat{n}_{i}^{a}=a_{i}^{\dagger} a_{i}, \hat{n}_{i}^{b}=b_{i}^{\dagger} b_{i}$. The sum $\sum_{\langle i, j\rangle}$ runs over all distinct pairs of first neighboring sites $i$ and $j, t_{a}\left(t_{b}\right)$ is the hopping integral between sites $i$ and $j$ for species $a(b)$, and $U_{a b}$ is the strength of the on-site interspecies repulsion.

We perform a quantum Monte Carlo study of the model (11) by using the Stochastic Green Function algorithm 40,41 with global space-time updates $\frac{42}{2}$ to solve the canonical ensemble on $L \times L$ lattices. We focus on the polarized phase diagram, with polarization $P=\frac{N_{b}-N_{a}}{L^{2}}$ and total density $\rho=\frac{N_{b}+N_{a}}{L^{2}}$, with $N_{a}$ and $N_{b}$ the number of heavy $a$ and light $b$ particles, respectively. For the other parameters we use the same values as in Ref. 34, namely $t_{a}=0.08 t, t_{b}=t$, and $U_{a b}=6 t$, where $t=1$.

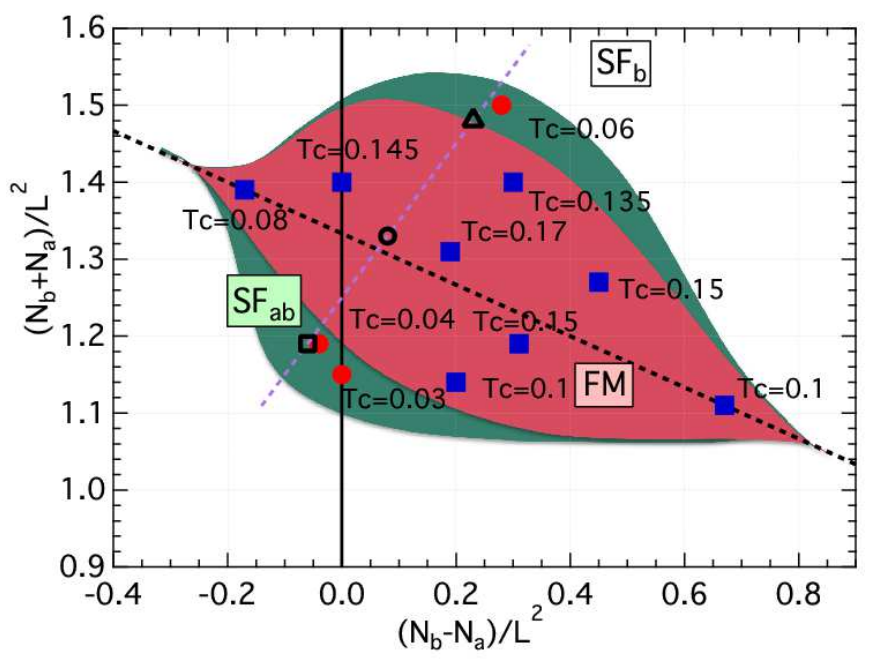

FIG. 1: (Color online) The total density, $\rho=\frac{N_{b}+N_{a}}{L^{2}}$, versus polarization, $P=\frac{N_{b}-N_{a}}{L^{2}}$, phase diagram at very low temperature. The transition temperatures associated with the data points are obtained from finite-size scaling calculations. The boundaries between the phases are estimated for $\beta t=60$ and $L=10$ with $t_{a}=0.08 t, t_{b}=1.00 t$, and $U_{a b}=6 t$. The boundaries may slightly change for the ground state. The red area shows the phase-separated ferromagnet (FM). The green area shows the region of superfluidity of both $a$ and $b$ species $\left(\mathrm{SF}_{a b}\right)$. The white region represents the superfluidity of light $b$ particles $\left(\mathrm{SF}_{b}\right)$ except that the system is an antiferromagnet at half-filling $(\rho=1$ and $P=0)$, and there is an antiferromagnetic to superfluid phase-separated region near half-filling $(1.0<\rho<1.1$ and $P=0)$ as discussed in Ref. 34 . Both particles are in a Mott insulating phase whenever their individual densities are integers (0 or 1$)$. The blue squares indicate the transition temperatures from the light species superfluid to the ferromagnetic phase. The red circles correspond to the transition temperatures from the light species superfluid to the phase where both species are superfluid. The black dotted line, $N_{b}+\frac{N_{a}}{2}=L^{2}$, follows the highest ferromagnetic critical temperature. The phase diagram as a function of temperature along this black dotted line is shown in Fig. 4. The momentum distributions shown in Fig. 6] are calculated along the purple dotted line $\left(N_{a}=0.625 L^{2}\right)$, which intersects the black dotted line. The momentum distributions for heavy and light species for three points along the purple dotted line are shown in the bottom panels of Fig. 6. The black line is the zero polarization axis.

\section{PHASE DIAGRAM AT LOW TEMPERATURE}

Figure 1 displays the total density $\rho$ versus polarization $P$ phase diagram at low temperature. In the thermodynamic limit, a ferromagnetic phase exists in a broad region of densities (red area), heavy $a$ and light $b$ particle superfluidity exists in a smaller region of densities (green area), and superfluidity of light $b$ particles (with heavy $a$ particles in the normal state) appears in most of the rest of the phase diagram (white area). Along the zero 
polarization axis there is an antiferromagnetic phase at half-filling ( $\rho=1$ and $P=0$ ), and an antiferromagnetic to superfluid phase-separated region for $1.0<\rho<1.1$ and $P=0$ as discussed in Ref. 34. The black dotted line, $N_{b}+\frac{N_{a}}{2}=L^{2}$, follows the highest ferromagnetic critical temperatures (optimal superfluid line). Along this line the system shows fully phase-separated regions of average local densities $n_{a} \sim 0$ together with $n_{b} \sim 1$, and $n_{a} \sim 1$ with $n_{b} \sim 0.5$. Therefore, the number of light particles, $N_{b}$, is given as $N_{b}=\left(L^{2}-N_{a}\right)+\frac{N_{a}}{2}$ (or $\frac{N_{a}}{2}+N_{b}=L^{2}$ ). In our previous study we did not distinguish between the phase where only the light particles are superfluid from the one where both species are superfluid. The ferromagnetic phase boundaries at zero polarization have also changed slightly.

The blue squares in Fig. 11indicate the transition temperature from the light species superfluid to the ferromagnetic phase for the given densities and polarizations. To find these transition temperatures we calculate the ferromagnetic susceptibility for different system sizes and perform a finite-size scaling. The susceptibility is given as $\chi(\mathbf{k})=\left\langle|\mathcal{A}(\mathbf{k})|^{2}\right\rangle-|\langle\mathcal{A}(\mathbf{k})\rangle|^{2}$ with

$$
\mathcal{A}(\mathbf{k})=\frac{1}{\beta} \int_{0}^{\beta} \sum_{j} e^{i \mathbf{k} \cdot \mathbf{r}_{j}}\left(n_{j}^{a}(\tau)-n_{j}^{b}(\tau)\right) d \tau .
$$

Following Ref. 43 we calculate the ferromagnetic susceptibility ratio, $\mathcal{R}$, defined as

$$
\mathcal{R}=\frac{\chi(0, \varepsilon)+\chi(0,-\varepsilon)+\chi(\varepsilon, 0)+\chi(-\varepsilon, 0)}{\chi(\varepsilon, \varepsilon)+\chi(-\varepsilon, \varepsilon)+\chi(\varepsilon,-\varepsilon)+\chi(-\varepsilon,-\varepsilon)}
$$

where $\varepsilon=\frac{2 \pi}{L}$. We impose all the point group symmetries in $\mathbf{k}$-space near $\mathbf{k} \sim 0$ to both the numerator and denominator to reduce the statistical noise associated with quantum Monte Carlo sampling.

The scaling behavior of the ferromagnetic transition temperature is shown in Fig. 2 where the susceptibility ratio $\mathcal{R}$ is plotted for different system sizes as a function of temperature $T / t$ at $\rho=1.4$ and $P=0$. In Fisher scaling $\underline{44}-\underline{46}$, the susceptibility at small wavenumber should scale as $\chi \sim L^{\frac{\gamma}{\nu}} g\left(L^{\frac{1}{\nu}}\left(T-T_{c}\right)\right)$, where $\gamma$ and $\nu$ are the critical exponents for the ferromagnetic susceptibility and correlation length, respectively. By looking at the ratio of the susceptibilities $\mathcal{R}$, the $L^{\frac{\gamma}{\nu}}$ factor is canceled. At the transition, the scaling function $g(0)$ is independent of $L$. Thus, the susceptibility ratio $\mathcal{R}$ versus temperature $T$ for different system sizes should cross at the critical temperature $\left(T=T_{c}=0.145 t\right)$ as it is shown in Fig. 2. By choosing the critical exponent of the correlation length as $\nu=1$, the value for a two-dimensional Ising transition, we find that the curves collapse onto one curve near the critical temperature (c.f., the inset of Fig. 21). If the transition is second order by considering symmetry arguments, it should belong to the Ising universality class. However, if we understand the polarized model as a Ising system within an external magnetic field, it is possible that the ferromagnetic transition is first order. Since it

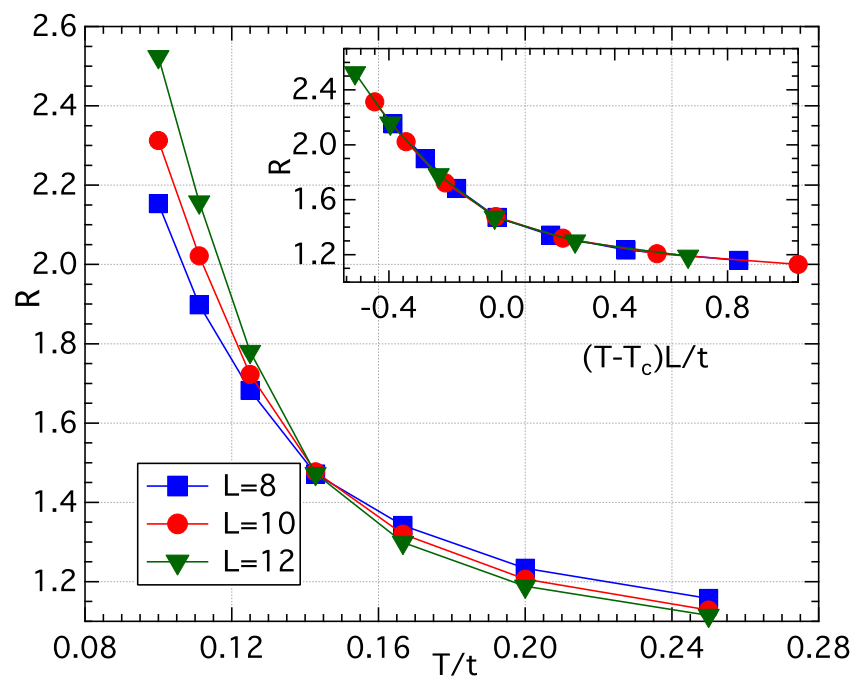

FIG. 2: (Color online) Scaling behavior of the ferromagnetic susceptibility for the continuous transition from light species superfluid to ferromagnet at $\rho=1.4$ and $P=0$. The susceptibility ratios, $\mathcal{R}$ (Eq. 3), versus temperature, $T / t$, for different system sizes cross at the critical temperature, $T_{c}=0.145 t$. The inset shows the scaling near the critical temperature. The curves collapse onto a single curve with the critical exponent of correlation length $\nu=1$. The data points are based on simulation results, the lines are guides to the eye.

is very difficult to distinguish between first and second order phase transitions with our finite size calculations, we can not clarify this issue.

The red circles in Fig. 1 indicate the superfluid transition temperature for the heavy species. The scaling behavior of the superfluid to normal liquid transition should follow that of the Kosterlitz-Thouless continuous transition. We note that the Hamiltonian (11) satisfies the condition (28) of Ref. 47, which allows one to relate the superfluid density to the fluctuations of the winding number $\stackrel{48}{ }$ In Fig. 3, we show the winding number of the $a$ particles, $\left\langle W^{2}\right\rangle$, as a function of temperature, $T / t$, for different system sizes. The order parameter, the superfluid density, has a universal jump of $\left\langle W^{2}\right\rangle=\frac{4}{\pi}$ at the critical point $\underline{\underline{49}}$ The black dotted line shows $\frac{4}{\pi} T$ as a function of temperature. We read the crossing temperature, $T_{L}$, for different system sizes. Then we use the relation between the crossing temperature, $T_{L}$, and the cluster size, $L, T_{L}-T_{c}(\infty) \propto \frac{1}{\ln ^{2}(L)}, \frac{50}{,}$ to find the critical temperature, $T_{c}$, in the thermodynamic limit. The inset of Fig. 3 displays this scaling. We find $T_{c}=0.03 t$ at $\rho=1.14$ and $P=0$.

\section{PHASE DIAGRAM ON THE OPTIMAL SUPERFLUID LINE}

To better understand the phases of the polarized model we investigate snapshots of the average local densities. 


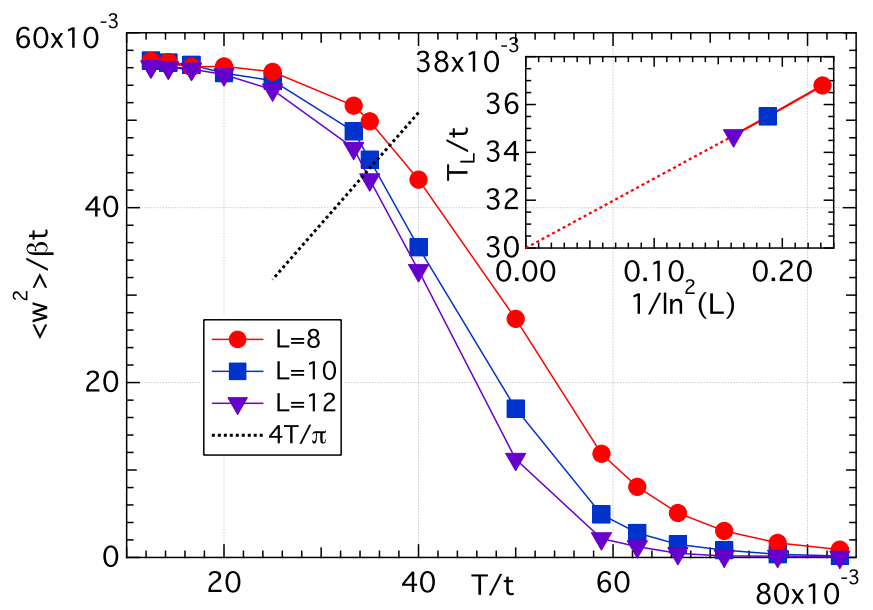

FIG. 3: (Color online) Winding number of heavy a particles as a function of temperature for different system sizes at $\rho=1.14$ and $P=0$. The black dotted line corresponds to $\frac{4 T}{\pi}$ and is used to find the crossing temperature for different system sizes. The inset shows the finite size scaling of the crossing temperatures to find the superfluid critical temperature, $T_{c}=$ $0.03 t$, in the thermodynamic limit. The data points are based on simulation results, the lines are guides to the eye.

From the snapshots we propose that superfluid and ferromagnetic states are optimal along the black dotted line shown in Fig. [1, where $\frac{N_{a}}{2}+N_{b}=L^{2}$, with $L^{2}$ the lattice size, and $N_{a}$ and $N_{b}$ the number of heavy and light atoms, respectively. Along this line the system shows fully phase-separated regions with average local densities $n_{a} \sim 0$ and $n_{b} \sim 1$ in the Mott region, and $n_{a} \sim 1$ and $n_{b} \sim 0.5$ in the Mott/superfluid region. The inset of Fig. 4 displays snapshots of these average local densities for heavy (left panel) and light (right panel) particles. Physically, this optimal line is driven by the fact that a superfluid with $n_{b} \sim 0.5$ gains the most energy per particle. As an example, for $N_{a}=50, N_{b}=75, L=10$ ( $\rho=1.25$ and $P=0.25$ ), half of the lattice is filled with $a$ particles and the other half with $b$ particles. The 25 remaining $b$ particles will occupy the region filled by $a$ particles and $n_{b}=\frac{25}{50}=0.5$ in that region. This reasoning is valid along this optimal superfluid line. However, when the system deviates far from $N_{a}=50 \%$ of the number of lattice sites, it is difficult to stabilize small and large phase-separated regions. In this case, the pattern may break. This also explains why the ferromagnetic phaseseparated phase is more stable for positive polarizations around $\rho=1.25$ and $P=0.25$. At half-filling of the heavy particles, this pattern is more stable since there are two large phase-separated regions reducing surface effects.

Fig. 4 shows the temperature, $T / t$, versus polarization, $P=\frac{N_{b}-N_{a}}{L^{2}}$, phase diagram on the optimal superfluid line, $\frac{N_{a}}{2}+N_{b}=L^{2}$. The blue squares are the ferromagnetic transition temperatures found by scaling as discussed in section [II. The red circles indicate the tran-

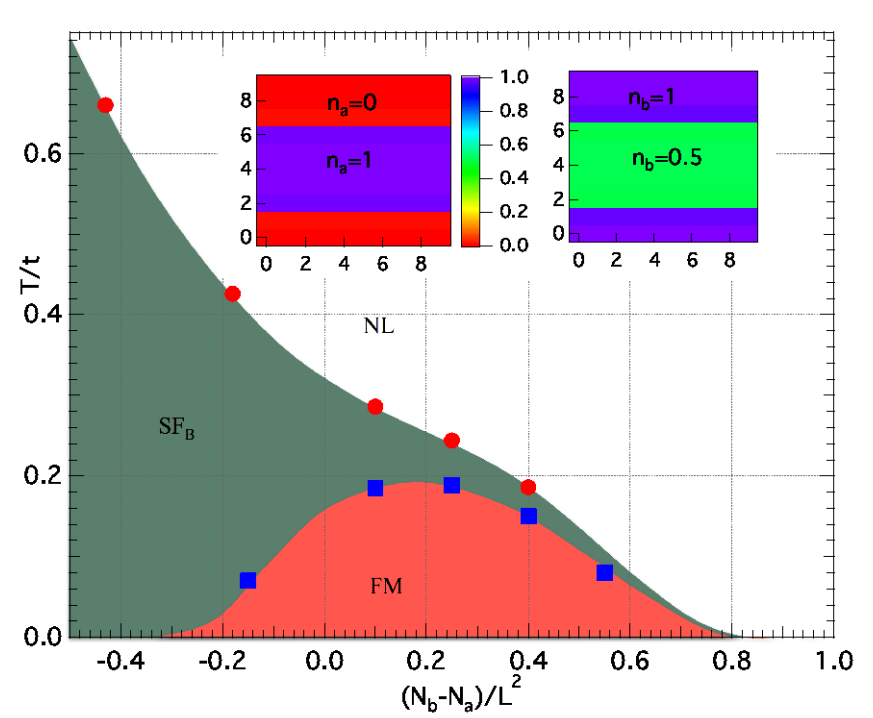

FIG. 4: (Color online) The temperature, $T / t$, versus polarization, $P=\frac{N_{b}-N_{a}}{L^{2}}$, phase diagram when $N_{b}+\frac{N_{a}}{2}=L^{2}$. The abscissa extends from $\rho=1, P=1\left(N_{a}=0, N_{b}=L^{2}\right)$ to $\rho=1.5, P=-0.5\left(N_{a}=L^{2}, N_{b}=L^{2} / 2\right)$. The orange area corresponds to the phase-separated ferromagnet (FM). The green area is the region where the light $b$ species displays superfluidity (SF). The white area is the normal liquid (NL). The blue squares and red circles indicating the boundaries between the phases are calculated by finite size scaling, see section III. The curves are guides to the eye. Since it is difficult to perform finite size scaling at very low temperatures, the edges of the phase diagram are estimations of the transition temperatures based on results of small clusters. The inset shows a snapshot of the average local densities versus lattice coordinates for $L=10, \rho=1.25, P=0.25$ and $\beta t=80$. Left panel: For $a$ particles, the red regions have $\left\langle n_{i}^{a}\right\rangle \sim 0$ while the occupation of the blue region is $\left\langle n_{i}^{a}\right\rangle \sim 1$. Right panel: For $b$ particles, the blue regions have $\left\langle n_{i}^{b}\right\rangle \sim 1$ while the occupation of the green region is $\left\langle n_{i}^{b}\right\rangle \sim 0.5$. The ferromagnetic phase separation occurs when the heavy species is in a Mott insulating state while the light one displays regions with either Mott insulating or superfluid behaviors.

sition temperatures for light species superfluid. Again, the scaling behavior of this light particle superfluid to normal liquid transition follows that of the KosterlitzThouless transition as discuss earlier for the heavy particles. In Fig. 囵, we show the winding of the $b$ particles, $\left\langle W^{2}\right\rangle$, as a function of temperature, $T / t$, for different system sizes. We find $T_{c}=0.245 t$ at $\rho=1.25$ and $P=0.25$ as shown in Fig 5

\section{MOMENTUM DISTRIBUTION}

A related experimentally accessible quantity that can distinguish different phases of bosons is the momentum 


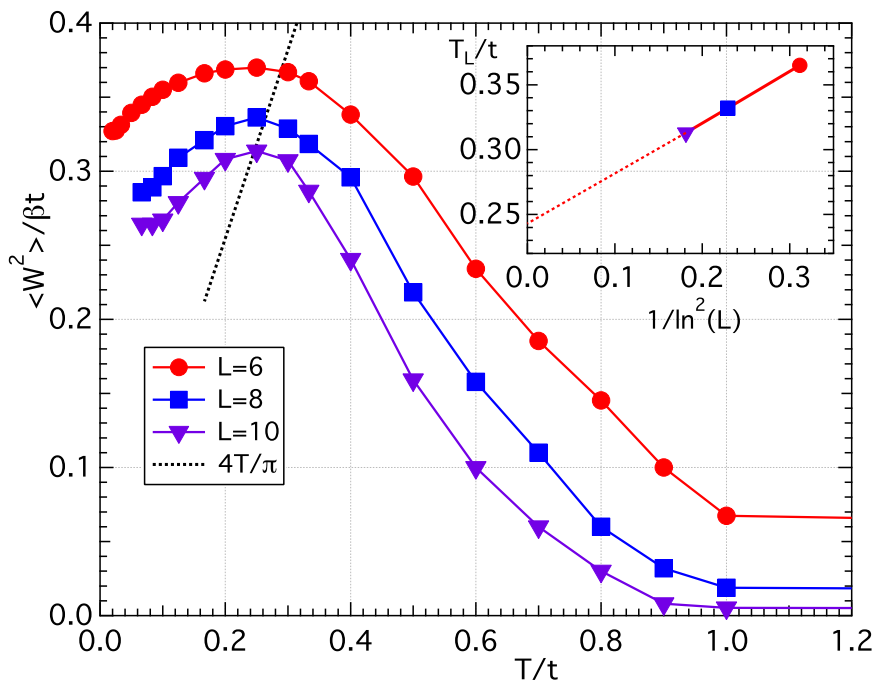

FIG. 5: (Color online) Winding number of light $b$ particles as a function of temperature for different system sizes at $\rho=1.25$ and $P=0.25$. The black dotted line shows $\frac{4 T}{\pi}$ and it is used to find the crossing temperatures for different system sizes. The lowering of the superfluid density at low temperatures occurs when the system enters the ferromagnetic phase where the light species displays both superfluid and Mott behaviors. The inset shows the finite size scaling of the crossing temperatures to find the superfluid critical temperature, $T_{c}=0.245 t$, in the thermodynamic limit for the continuous transition. The data points are based on simulation results, the lines are guides to the eye.

distribution. It is defined as

$$
N(\mathbf{k})=\frac{1}{L^{2}} \sum_{k, l} e^{i \mathbf{k} \cdot\left(\mathbf{r}_{k}-\mathbf{r}_{l}\right)}\left\langle a_{k}^{\dagger} a_{l}\right\rangle,
$$

with the momentum $k_{x, y}=\frac{2 \pi}{L} m, m=0,1, \ldots, L-1$. The superfluid ground state is characterized by a peak at zero momentum, $\mathbf{k}=(0,0)$, while the Mott insulator phase has an uniform momentum distribution 13,23 Fig. 6] displays the momentum distribution of heavy and light particles for the ferromagnetic and superfluid phases along the purple dotted line in Fig. 1. The momentum distribution at zero wavevector, $\mathbf{k}=(0,0)$, and $\beta t=50$ as a function of polarization for heavy and light species is shown in the top panel. The momentum distribution of the heavy particles at zero momentum is small in the ferromagnetic region but large in the superfluid phase. The light particles show significantly less variation with polarization and have a value which is consistently large compared to the ferromagnetic state of the heavy particles, indicative of a superfluid state. The momentum distributions for heavy and light species for three points along the purple dotted line are shown in the bottom panels. The left panels show that for $\rho=1.19$ and $P=-0.06$, heavy and light distributions display peaks at $\mathbf{k}=(0,0)$ indicating superfluidity of both species. The same happens at the right panels for $\rho=1.48$ and $P=0.23$. The middle panel at $\rho=1.33$ and $P=0.08$ displays a $\mathbf{k}=(0,0)$ peak

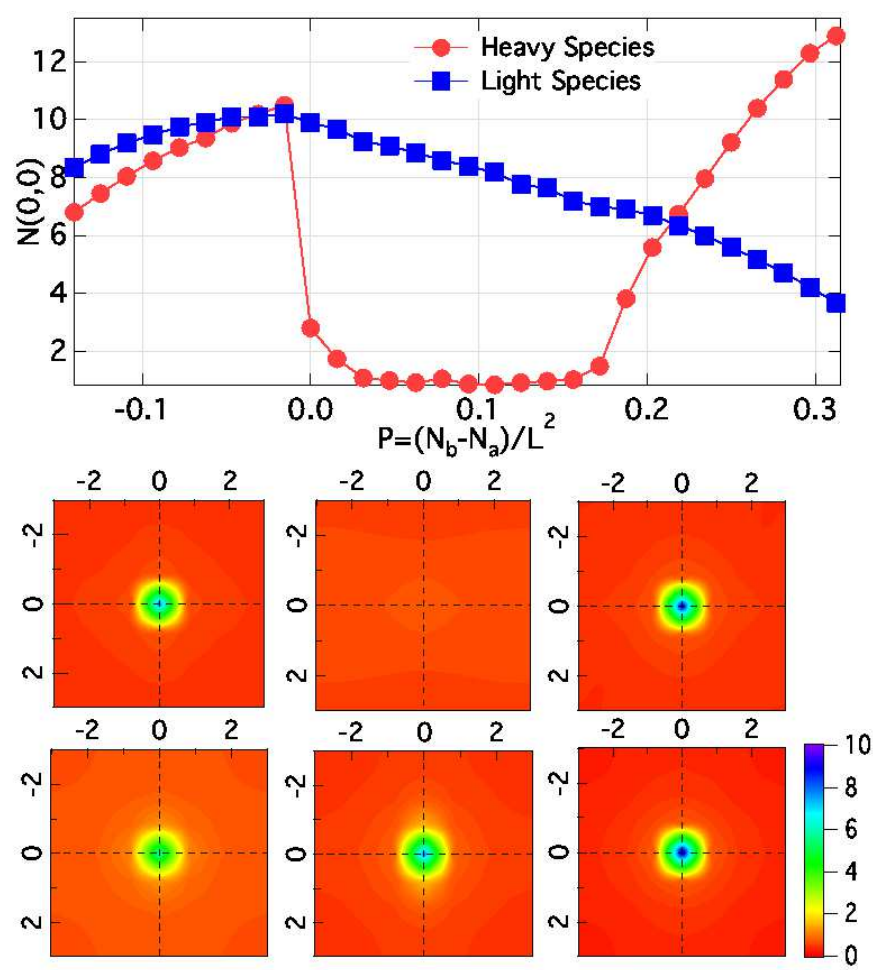

FIG. 6: (Color online) The $\mathbf{k}$-space momentum distribution for $\beta t=50$ and $L=8$. Top panel: The momentum distribution at zero momentum $\mathbf{k}=(0,0)$ as a function of polarization for heavy (red circles) and light (blue squares) species along the purple dotted line shown in Fig. 1 The data points are based on simulation results, the lines are guides to the eye. Bottom panels: The momentum distributions for heavy and light species for three points along the purple dotted line shown in Fig. 1. Left panel: The momentum distribution at $\rho=1.19$ and $P=-0.06$ (open black square in Fig. 1). For both $b$ (bottom) and $a$ (top) particles, the distributions have a peak at $\mathbf{k}=(0,0)$ which corresponds to superfluid behavior. Middle panel: The momentum distribution for the ferromagnetic phase at $\rho=1.33$ and $P=0.08$ (open black circle in Fig. 1). For the $b$ particles (bottom), the distribution has a peak at $\mathbf{k}=(0,0)$ which corresponds to superfluid behavior. For a particles (top), the distribution is uniform corresponding to Mott behavior. Right panel: The momentum distribution at $\rho=1.48$ and $P=0.23$ (open black triangle in Fig. 1). For both $b$ (bottom) and $a$ (top) particles, the distributions have a peak at $\mathbf{k}=(0,0)$ corresponding to superfluid behavior.

in the momentum distribution of the light species while the momentum distribution of heavy particles is uniform. This behavior is consistent with the phase-separated ferromagnetic phase where the heavy species a becoming Mott while the light one $b$ displays regions with Mott insulating and superfluid behaviors. 


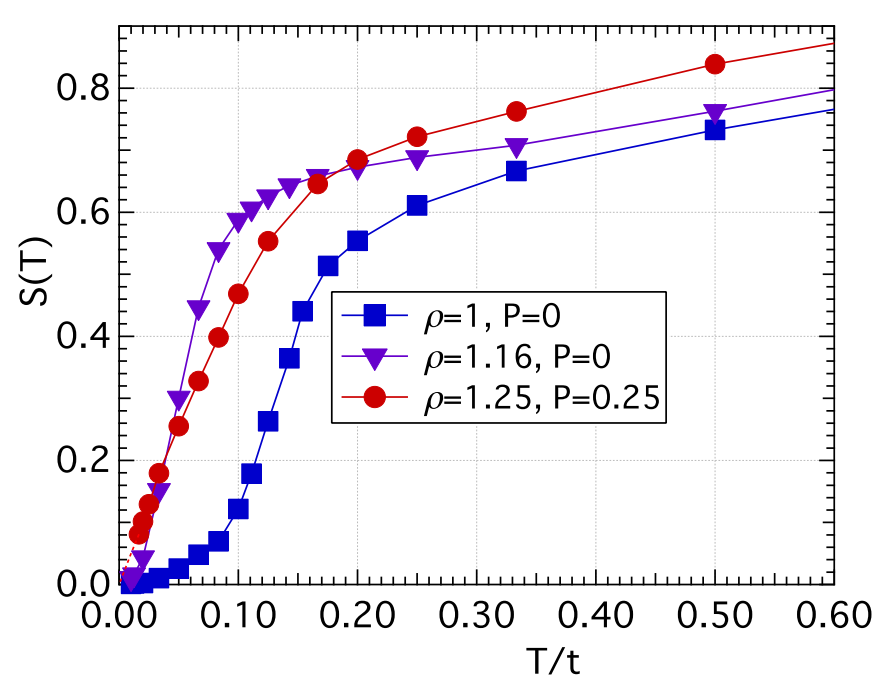

FIG. 7: (Color online) Entropy, $S(T)$, for $L=10, t_{a}=0.08$, $t_{b}=t=1$, and $U_{a b}=6$, as a function of temperature, $T / t$, for three different combinations of total densities, $\rho$, and polarizations, $P$. The red circles show the entropy of the phaseseparated ferromagnetic phase at $\rho=1.25$ and $P=0.25$. The blue squares display the entropy of the antiferromagnetic phase at $\rho=1$ and $P=0$. The purple triangles show the entropy of the superfluid phase at $\rho=1.16$ for the non-polarized system. The data points are based on simulation results, the lines are guides to the eye.

\section{ENTROPY}

Reaching the low entropies and temperatures required to observe magnetically ordered or Mott insulating phases is still experimentally challenging. In the ferromagnetic phase separated region, the superfluid ordering of light $b$ particles can carry most of the entropy, leaving the entropy of the heavy species in this phase essentially zero. Thus the ferromagnetic phase-separated phase can have large entropy. Fig. 7 shows the entropy for an $L=10$ system calculated following Ref. 51 for three different densities and polarizations, $\rho=1$ and $P=0$ (antiferromagnet), $\rho=1.16$ and $P=0$ (superfluid), and $\rho=1.25$ and $P=0.25$ (ferromagnet). The entropy of the ferromagnetic phase is greater than that of the antiferromagnetic phase and similar to that of the superfluid state, especially for low temperatures, indicating that it may be more accessible experimentally. The entropy, $S(T)$ is calculated by integrating the internal energy per site, $E(T)$, as:

$$
\mathcal{S}(\beta, n)=S(0, n)+\beta E(\beta, n)-\int_{0}^{\beta} E\left(\beta^{\prime}, n\right) d \beta^{\prime},
$$

where $S(0, n)$ depends on the possible per site occupation of $a$ and $b$ particles.

\section{CONCLUSION}

By introducing a population imbalance between the two species, we find an extended region of phaseseparated ferromagnetism in the two-dimensional twospecies hard-core bosonic Hubbard model. The average local densities show that the heavy species has Mottinsulating behavior while the light species is phase separated into both Mott insulating and superfluid regions. This phase exists for a broad range of temperatures and polarizations. In this polarized model we find the optimal superfluid line, $\frac{N_{a}}{2}+N_{b}=L^{2}$, where the system shows high transition temperatures and fully phase-separated regions at low temperatures with average local densities $n_{a} \sim 0$ and $n_{b} \sim 1$ on one of the regions, and $n_{a} \sim 1$, $n_{b} \sim 0.5$ on the other. This line exists because the superfluidity of light species with $n_{b} \sim 0.5$ gains the most energy per particle. Further the ferromagnetic phaseseparated phase is more stable for positive polarizations around $\rho=1.25$ and $P=0.25$. When the system deviates far from half-filling of the heavy a particles, the ferromagnetic phase vanishes since it is difficult to stabilize small and large phase-separated regions. By using finite-size scaling of ferromagnetic susceptibility ratios, we find the correlation length exponent $\nu \approx 1$ which is consistent with a two-dimensional Ising ferromagnet. Despite its ferromagnetic order, this phase has relatively high global entropy, which suggests that its experimental observation in cold atoms should be more accessible.

\section{ACKNOWLEDGMENT}

This work is supported by NSF OISE-0952300 (KH, VGR and JM) and DMR-1237565 (KH and JM). Additional support was provided by the NSF EPSCoR Cooperative Agreement No. EPS-1003897 with additional support from the Louisiana Board of Regents (KMT and $\mathrm{MJ})$. This work used the Extreme Science and Engineering Discovery Environment (XSEDE), which is supported by the National Science Foundation grant number ACI1053575, and the high performance computational resources provided by the Louisiana Optical Network Initiative (http://www.loni.org).
1 S. Yunoki, J. Hu, A. L. Malvezzi, A. Moreo, N. Furukawa and E. Dagotto,Phys. Rev. Lett. 80, 845(1998).

2 A. Moreo, S. Yunoki and E. Dagotto, Science 2034, 283
(1999).

3 M. Uehara, S. Mori, C. H. Chen, S. -W. Cheong, Nature 399, 560 (1999). 
${ }^{4}$ E. Dagotto, T. Hotta and A. Moreo, Phys. Rep. 344, 1-153 (2001).

5 P. Coleman and A. J. Schofield, Nature, 433, 227 (2005).

${ }^{6}$ Q. Si and F. Steglich, Science 329, 1161 (2010).

7 E. Dagotto, Science 309, 257 (2005).

8 D. Jaksch, C. Bruder, J. I. Cirac, C. W. Gardiner, and P. Zoller, Phy. Rev. Lett. 81, 3108 (1998).

9 W. Hofstetter, J. I. Cirac, P. Zoller, E. Demler, and M. D. Lukin, Phys. Rev. Lett. 89, 220407 (2002).

10 T. Esslinger, Annual Review of Condensed Matter Physics 1, 129 (2010).

11 E. Timmermans, P. Tommasini, M. Hussein, A. Kerman, Phys. Rep. 315, 199, (1999).

12 T. Köhler, K. Góral, and P. S. Julienne, Rev. Mod. Phys. 78, 1311, (2006).

13 M. Greiner, O. Mandel, T. Esslinger, T. W. Hänsch, and I. Bloch, Nature (London), 415, 39 (2002).

14 M. P. A. Fisher, P. B. Weichman, G. Grinstein, and D. S. Fisher, Phys. Rev. B 40, 546 (1989).

15 G. G. Batrouni and R. T. Scalettar, Phys. Rev. Lett. 84, 1599 (2000).

16 F. Schreck, L. Khaykovich, K. L. Corwin, G. Ferrari, T. Bourdel, J. Cubizolles, and C. Salomon, Phys. Rev. Lett. 87, 080403 (2001).

17 A. Albus, F. Illuminati, and J. Eisert, Phys. Rev. A 68, 023606 (2003).

18 G. Modugno, L. Khaykovich, K. L. Corwin, G. Ferrari, T. Bourdel, J. Cubizolles, and C. Salomon, Science, 297 (2002).

19 C. Ospelkaus, S. Ospelkaus, K. Sengstock, and K. Bongs, Phys. Rev. Lett. 96, 020401 (2006).

${ }^{20}$ G. Roati, M. Zaccanti, C. D'Errico, J. Catani, M. Modugno, A. Simoni, M. Inguscio, and G. Modugno, Phys. Rev. Lett. 99, 010403 (2007).

21 G. Thalhammer, G. Barontini, L. De Sarlo, J. Catani, F. Minardi, and M. Inguscio, Phys. Rev. Lett. 100, 210402 (2008).

22 S. B. Papp, J. M. Pino, and C. E. Wieman, Phys. Rev. Lett. 101, 040402 (2008).

23 J. Catani, L. De Sarlo, G. Barontini, F. Minardi, and M. Inguscio, Phys. Rev. A 77, 011603 (2008).

${ }^{24}$ M. Taglieber, A.-C. Voigt, T. Aoki, T. W. Hänsch, and K. Dieckmann, Phys. Rev. Lett. 100, 010401 (2008).

25 S. Taie, Y. Takasu, S. Sugawa, R. Yamazaki, T. Tsujimoto, R. Murakami, and Y. Takahashi, Phys. Rev. Lett. 105, 190401 (2010).

${ }^{26}$ E. Altman, W. Hofstetter, E. Demler, and M. D. Lukin, New J. Phys. 5, 113 (2003).

27 S. G. Söyler, B. Capogrosso-Sansone, N. V. Prokof'ev, and
B. V. Svistunov, New J. Phys. 11, 073036 (2009).

28 B. Capogrosso-Sansone, S. G. Söyler, N. V. Prokof'ev, and B. V. Svistunov, Phys. Rev. A 81, 053622 (2010).

29 S. Powell, Phys. Rev. A 79, 053614 (2009).

30 A. Sotnikov, D. Cocks, and W. Hofstetter, Phys. Rev. Lett. 109, 065301 (2012).

31 Y. Kuno, K. Suzuki, and I. Ichinose, J. Phys. Soc. Jpn 82, 12450 (2013).

${ }^{32}$ F. Trousselet, P. Rueda-Fonseca, and A. Ralko, Phys. Rev. B 89085104 (2014).

33 J. P. Lv, Q. H. Chen, and Y. Deng, Phys. Rev. A 89, 013628 (2014).

${ }^{34}$ K. Hettiarachchilage, V. G. Rousseau, K.-M. Tam, M. Jarrell, and J. Moreno, Phys. Rev. B 88, 161101(R) (2013).

35 C. Monroe, D. M. Meekhof, B. E. King, S. R. Jefferts, W. M. Itano, D. J. Wineland, and P. Gould, Phys. Rev. Lett. 75, 4011 (1995).

36 M. Popp, J.-J. Garcia-Ripoll, K. G. Vollbrecht, and J. I. Cirac, Phys. Rev. A 74, 013622 (2006).

37 X. Li, T. A. Corcovilos, Y. Wang, and D. S. Weiss, Phys. Rev. Lett. 108, 103001 (2012).

38 T.-L. Ho and Q. Zhou, Proc. Natl. Acad. Sci. USA 106, 6916 (2009).

${ }^{39}$ K. Pilch, A. D. Lange, A. Prantner, G. Kerner, F. Ferlaino, H.-C. Nägerl, and R. Grimm, Phys. Rev. A 79, 042718 (2009).

40 V. G. Rousseau, Phys. Rev. E 77, 056705 (2008).

41 V. G. Rousseau, Phys. Rev. E 78, 056707 (2008).

42 V. G. Rousseau and D. Galanakis, arXiv:1209.0946 (2012).

43 R. A. Baños, A. Cruz, L.A. Fernandez, J. M. Gil-Narvion, A. Gordillo-Guerrero, M. Guidetti, D. Iñiguez, A. Maiorano, E. Marinari, V. Martin-Mayor, J. Monforte-Garcia, A. Muñoz Sudupe, D. Navarro, G. Parisi, S. Perez-Gaviro, J. J. Ruiz-Lorenzo, S.F. Schifano, B. Seoane, A. Tarancon, P. Tellez, R. Tripiccione, D. Yllanes, Proc. Natl. Acad. Sci. U.S.A. 109, 6452 (2012).

44 M. E. Fisher, Rep. Prog. Phys. 30, 615 (1967).

45 M. E. Fisher, Rev. Mod. Phys. 46, 597 (1974).

46 M. E. Fisher, Rev. Mod. Phys. 70, 653 (1998).

47 V. G. Rousseau, Phys. Rev. B 90, 134503 (2014).

48 E. L. Pollock and D. M. Ceperley, Phys. Rev. B 36, 8343 (1987).

49 D. R. Nelson and J. M. Kosterlitz, Phys. Rev. Lett. 39, 1201 (1977).

50 M. Boninsegni and N. Prokof'ev, Phys. Rev. Lett. 95, 237204 (2005).

51 F. Werner, O. Parcollet, A. Georges, and S. R. Hassan, Phys. Rev. Lett. 95, 056401 (2005). 